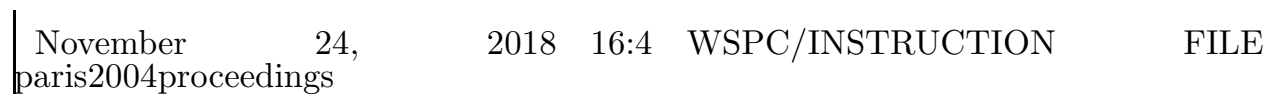

International Journal of Modern Physics A

(C) World Scientific Publishing Company

\title{
THE PENTAQUARKS IN THE LINEAR MOLECULAR HEPTAQUARK MODEL
}

\author{
P. Bicudo \\ Dep. Física and CFIF, Instituto Superior Técnico, Av. Rovisco Pais, 1049-001 Lisboa, Portugal, \\ email : bicudo@ist.utl.pt
}

\begin{abstract}
In this talk, multiquarks are studied microscopically in a standard quark model. In pure ground-state pentaquarks the short-range interaction is computed and it is shown to be repulsive. An additional quark-antiquark pair is then considered, and this is suggested to produce linear molecular system, with a narrow decay width. The quarks assemble in three hadronic clusters, and the central hadron provides stability. The possible crypto-heptaquark hadrons with exotic pentaquark flavours, with strange, charmed and bottomed quarks, are predicted.
\end{abstract}

Exotic multiquarks are expected since the early works of Jaffe ${ }^{1}$, and the masses and decays in the SU(3) exotic anti-decuplet were first predicted within the chiral soliton model 2 . The pentaquarks have been revived recently by several searches of the $\Theta^{+}(1540) 34567891011121314151617181920$, first discovered at LEPS 3 , and by searches of the $\Xi^{--}(1860) 212223$ and of the $D^{*-} p(3100)^{24}$, observed respectively at NA49 21 and at $\mathrm{H}^{24}$. Pentaquark structures have also been studied on the lattice 252627282930313233. Moreover multiquarks are favoured by the presence of several different flavours 363738 . The observation of the $D^{*-} p(3100)$ at $\mathrm{H} 1$, the observation of double-charmed baryons at SELEX 34 , and the future search of double-charmed baryons at COMPASS 35 suggest that new pentaquarks with one or two heavy quarks remain to be discovered.

It is well known that a narrow pentaquark must contain an excitation, to prevent a decay width of hundreds of $\mathrm{MeV}$ to a meson-baryon channel. Here I consider that a s-wave flavour-singlet light quark-antiquark pair $l \bar{l}$ is added to the pentaquark $M$. The resulting heptaquark $M^{\prime}$ is a state with parity opposite to the original $M[39$, due to the intrinsic parity of fermions and anti-fermions. The ground-state of $M^{\prime}$ is also naturally rearranged in a s-wave baryon and in two s-wave mesons, where the two outer hadrons are repelled, while the central hadron provides stability. Because the s-wave pion is the lightest hadron, the minimum energy needed to create a quark-antiquark pair can be as small as 100-200 MeV. This energy shift is lower than the typical energy of $300-600 \mathrm{MeV}$ of spin-isospin or angular excitations in hadrons. Moreover, the heptaquarks $M^{\prime}$ low-energy p-wave decay (after the extra quark-antiquark pair is annihilated), results in a very narrow decay width, consistent with the observed exotic flavour pentaquarks.

Recently this principle was used to suggest that the $\Theta^{+}(1540)$ is a $K \bullet \pi \bullet N$ 


\section{$\begin{array}{llllll}\text { November } & 24, & 2018 & 16: 4 & \text { WSPC/INSTRUCTION } & \text { FILE }\end{array}$} paris2004proceedings

2 P. Bicudo

Table 1. Exotic-flavour pentaquarks with no heavy quark.

\begin{tabular}{cccc}
\hline flavour & linear molecule & mass $[\mathrm{GeV}]$ & decay channels \\
\hline$I=1 / 2, \operatorname{ssss} \bar{l}(+3 l \bar{l}):$ & five-hadron molecule & & \\
\hline$I=1, \operatorname{sss} l \bar{l}(+2 l \bar{l}):$ & four-hadron molecule & & \\
\hline$I=3 / 2, \operatorname{ssll} \bar{l}(+l \bar{l})=$ & $s \bar{l} \bullet l l l \bullet s \bar{l}:$ & & \\
& $\bar{K} \bullet N \bullet \bar{K}=\mathbf{\Xi}^{--}$ & 1.86 & $\bar{K}+\Sigma, \pi+\Xi$ \\
\hline$I=2, \operatorname{slll} \bar{l}(+l \bar{l})=$ & $s \bar{l} \bullet l l l \bullet l \bar{l}:$ & pion unbound & \\
\hline$I=5 / 2, \operatorname{llll} \bar{l}(+l \bar{l})=$ & $l \bar{l} \bullet l l l \bullet l \bar{l}:$ & pion unbound & \\
\hline$I=0, \operatorname{llll} \bar{s}(+l \bar{l})=$ & $l \bar{s} \bullet l \bar{l} \bullet l l l:$ & & $K+N$ \\
\hline
\end{tabular}

molecule with binding energy of $30 \mathrm{MeV} 404142$, and the $\Xi^{--}(1862)$ is a $\bar{K} \bullet N \bullet \bar{K}$ molecule with a binding energy of $60 \mathrm{MeV} 4043$. I also suggest that the new positive parity scalar $D_{s}(2320)$ and axial $D_{s+}(2460)$ are $\bar{K} \bullet D$ and $\bar{K} \bullet D^{*}$ multiquarks 44 , and that the $D^{*-} p(3100)$ is consistent with a $D^{*} \bullet \pi \bullet N$ linear molecule with an energy of $15 \mathrm{MeV}$ above threshold 4045 . I now predict new exotic strange, charmed and bottomed pentaquarks compatible with the linear molecular heptaquark model.

In this talk, multiquarks are studied microscopically in a standard quark-model (QM) Hamiltonian. The energy of the multiquark state, and the short range interaction of the mesonic or baryonic subclusters of the multiquark are both computed with the multiquark matrix element of the QM Hamiltonian,

$$
H=\sum_{i} T_{i}+\sum_{i<j} V_{i j}+\sum_{i \bar{j}} A_{i \bar{j}} .
$$

Each quark or antiquark has a kinetic energy $T_{i}$. The colour-dependent two-body interaction $V_{i j}$ includes the standard QM confining and hyperfine terms,

$$
V_{i j}=\frac{-3}{16} \vec{\lambda}_{i} \cdot \vec{\lambda}_{j}\left[V_{\text {conf }}(r)+V_{\text {hyp }}(r) \vec{S}_{i} \cdot \vec{S}_{j}\right] .
$$

The potential of eq. (2) reproduces the meson and baryon spectrum with quark and antiquark bound states (from heavy quarkonium to the light pion mass). Moreover, the Resonating Group Method (RGM) 46 was applied by Ribeiro 47 to show that in exotic $N+N$ scattering the quark two-body-potential, together with the Pauli repulsion of quarks, explains the $N+N$ hard core repulsion. Recently, addressing a tetraquark system with $\pi+\pi$ quantum numbers, it was shown that the QM with the quark-antiquark annihilation $A_{i \bar{j}}$ also fully complies with chiral symmetry, including the Adler zero and the Weinberg theorem 4849150.

For the purpose of this talk, only the matrix elements of the potentials in eq. (11) matter. The hadron spectrum constrains the hyperfine potential

$$
\left\langle V_{\text {hyp }}\right\rangle \simeq \frac{4}{3}\left(M_{\Delta}-M_{N}\right) \simeq M_{K^{*}}-M_{K} .
$$




\section{$\begin{array}{lllll}\text { November } & 24, & 2018 & 16: 4 & \text { WSPC/INSTRUCTION }\end{array}$} paris2004proceedings

The Pentaquarks in the Linear Molecular Heptaquark Model 3

Table 2. Exotic flavour pentaquarks with one heavy quark.

\begin{tabular}{|c|c|c|c|}
\hline flavour & linear molecule & $\operatorname{mass}[\mathrm{GeV}]$ & decay channels \\
\hline$I=1 / 2, H \operatorname{sss} \bar{l}(+2 l \bar{l}):$ & four-hadron molecule & & \\
\hline$I=1, H s s \bar{l}(+l \bar{l})=$ & $\begin{array}{l}s \bar{l} \bullet l l H \bullet s \bar{l}: \\
\bar{K} \bullet \Lambda_{c} \bullet \bar{K} \\
\bar{K} \bullet \Lambda_{b} \bullet \bar{K}\end{array}$ & $\begin{array}{l}3.23 \pm 0.03 \\
6.57 \pm 0.03\end{array}$ & $\begin{array}{l}\bar{K}+\Xi_{c}, \pi+\Omega_{c} \\
\bar{K}+\Xi_{b}, \pi+\Omega_{b}\end{array}$ \\
\hline$I=3 / 2, H \operatorname{sll} \bar{l}(+l \bar{l})=$ & $\begin{array}{c}s \bar{l} \bullet l l l \bullet H \bar{l}: \\
\bar{K} \bullet N \bullet D \\
\bar{K} \bullet N \bullet D^{*} \\
\bar{K} \bullet N \bullet \bar{B} \\
\bar{K} \bullet N \bullet \bar{B}^{*}\end{array}$ & $\begin{array}{l}3.25 \pm 0.03 \\
3.39 \pm 0.03 \\
6.66 \pm 0.03 \\
6.71 \pm 0.03\end{array}$ & $\begin{array}{c}\bar{K}+\Sigma_{c}, D+\Sigma, \pi+\Xi_{c} \\
\bar{K}+\Sigma_{c}, D^{*}+\Sigma, \pi+\Xi_{c} \\
\bar{K}+\Sigma_{b}, \bar{B}+\Sigma, \pi+\Xi_{b} \\
\bar{K}+\Sigma_{b}, \bar{B}^{*}+\Sigma, \pi+\Xi_{b}\end{array}$ \\
\hline$I=2, \operatorname{Hlll} \bar{l}(+l \bar{l})=$ & $l \bar{l} \bullet l l l \bullet H \bar{l}:$ & pion unbound & \\
\hline $\begin{array}{l}I=1 / 2, \operatorname{Hlll} \bar{s}(+l \bar{l})= \\
I=1 / 2, \operatorname{Hlll} \bar{s}(+l \bar{l})=\end{array}$ & $\begin{array}{c}l \bar{s} \bullet l \bar{l} \bullet l l H: \\
K \bullet \pi \bullet \Sigma_{c} \\
K \bullet \pi \bullet \Sigma_{b} \\
l \bar{s} \bullet H \bar{l} \bullet l l l: \\
K \bullet \bar{D} \bullet N \\
K \bullet \bar{D}^{*} \bullet N \\
K \bullet \bar{B} \bullet N \\
K \bullet \bar{B}^{*} \bullet N\end{array}$ & $\begin{array}{l}3.08 \pm 0.03 \\
6.41 \pm 0.1 \\
3.25 \pm 0.03 \\
3.39 \pm 0.03 \\
6.66 \pm 0.03 \\
6.71 \pm 0.03\end{array}$ & 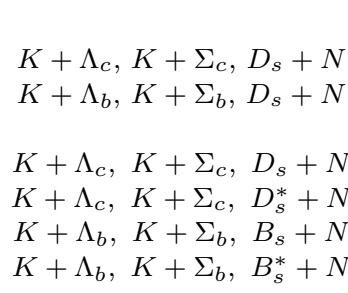 \\
\hline
\end{tabular}

From the pion mass 51 , the matrix elements of the annihilation potential are,

$$
\langle A\rangle_{S=0} \simeq-\frac{2}{3}\left(2 M_{N}-M_{\Delta}\right)
$$

which is correct for the annihilation of $u$ or $d$ quarks.

The annihilation potential only shows up in non-exotic channels, and it is clear from eq. (44) that the annihilation potential provides an attractive (negative) interaction. The quark-quark(antiquark) potential is dominated by the interplay of the hyperfine interaction of eq. (3) and the Pauli quark exchange. In s-wave systems with low spin this results in a repulsive interaction. Therefore, I arrive at the attraction/repulsion criterion for groundstate hadrons:

- whenever the two interacting hadrons have quarks (or antiquarks) with a common flavour, the repulsion is increased by the Pauli principle;

- when the two interacting hadrons have a quark and an antiquark with the same flavour, the attraction is enhanced by the quark-antiquark annihilation.

For instance, $u u d-s \bar{u}$ is attractive, and $u u d-u \bar{s}$ is repulsive. This qualitative rule is confirmed by quantitative computations of the short-range interactions of the $\pi, N, K, D, D^{*}, B, B^{*} 40444543484950$.

Again, the attraction/repulsion criterion shows that the exotic pentaquarks containing five quarks only are repelled. To increase binding we include a light $l \bar{l}$ quarkantiquark pair in the system. I now detail the strategy to find the possible linear heptaquark molecules.

a) The top quark is excluded because it is too unstable. To minimise the short-range 


\section{November 24, $2018 \quad 16: 4 \quad$ WSPC/INSTRUCTION $\quad$ FILE}

paris2004proceedings

$4 \quad$ P. Bicudo

Table 3. Exotic flavour pentaquarks with one heavy anti-quark.

\begin{tabular}{|c|c|c|c|}
\hline flavour & linear molecule & $\operatorname{mass}[\mathrm{GeV}]$ & decay channels \\
\hline$I=0, \operatorname{ssss} \bar{H}(+3 l \bar{l}):$ & five-hadron molecule & & \\
\hline$I=1 / 2, \operatorname{sssl} \bar{H}(+2 l \bar{l})$ & four-hadron molecule & & \\
\hline$I=0, s s l l \bar{H}(+l \bar{l})=$ & $\begin{array}{c}l \bar{H} \bullet l \bar{l} \bullet l s s \\
\bar{D} \bullet \pi \bullet \Xi \\
\bar{D}^{*} \bullet \pi \bullet \Xi \\
B \bullet \pi \bullet \Xi \\
B^{*} \bullet \pi \bullet \Xi\end{array}$ & $\begin{array}{l}3.31 \pm 0.03 \\
3.45 \pm 0.03 \\
6.73 \pm 0.03 \\
6.77 \pm 0.03\end{array}$ & $\begin{array}{c}\bar{D}+\Xi, \bar{D}_{s}+\Lambda \\
\bar{D}^{*}+\Xi, \bar{D}_{s}^{*}+\Lambda, \bar{D}_{s}+\Lambda \\
B+\Xi, B_{s}+\Lambda \\
B^{*}+\Xi, B_{s}^{*}+\Lambda, B_{s}+\Lambda\end{array}$ \\
\hline$I=1 / 2, \operatorname{slll} \bar{H}(+l \bar{l})=$ & $\begin{array}{c}l \bar{H} \bullet l \bar{l} \bullet l l s \\
\bar{D} \bullet \pi \bullet \Sigma \\
\bar{D}^{*} \bullet \pi \bullet \Sigma \\
B \bullet \pi \bullet \Sigma \\
B^{*} \bullet \pi \bullet \Sigma \\
l \bar{H} \bullet s \bar{l} \bullet l l l \\
\bar{D} \bullet \bar{K} \bullet N \\
\bar{D}^{*} \bullet \bar{K} \bullet N \\
B \bullet \bar{K} \bullet N \\
B^{*} \bullet \bar{K} \bullet N\end{array}$ & $\begin{array}{l}3.19 \pm 0.03 \\
3.33 \pm 0.03 \\
6.60 \pm 0.03 \\
6.64 \pm 0.03 \\
3.25 \pm 0.03 \\
3.39 \pm 0.03 \\
6.66 \pm 0.03 \\
6.71 \pm 0.03\end{array}$ & $\begin{array}{c}\bar{D}+\Lambda, \bar{D}+\Sigma, \bar{D}_{s}+N \\
\bar{D}^{*}+\Lambda, \bar{D}^{*}+\Sigma, \bar{D}_{s}^{*}+N \\
B+\Lambda, B+\Sigma, B_{s}+N \\
B^{*}+\Lambda, B^{*}+\Sigma, B_{s}^{*}+N \\
\\
\bar{D}+\Lambda, \bar{D}+\Sigma, \bar{D}_{s}+N \\
\bar{D}^{*}+\Lambda, \bar{D}^{*}+\Sigma, \bar{D}_{s}^{*}+N \\
B+\Lambda, B+\Sigma, B_{s}+N \\
B^{*}+\Lambda, B^{*}+\Sigma, B_{s}^{*}+N\end{array}$ \\
\hline$I=0, l l l l \bar{H}(+l \bar{l})=$ & $\begin{array}{c}l \bar{H} \bullet l \bar{l} \bullet l l l \\
\bar{D} \bullet \pi \bullet N \\
\bar{D}^{*} \bullet \pi \bullet N=\overline{\mathbf{D}}^{*-} \mathbf{p} \\
B \bullet \pi \bullet N \\
B^{*} \bullet \pi \bullet N\end{array}$ & $\begin{array}{l}2.93 \pm 0.03 \\
\quad 3.10 \\
6.35 \pm 0.03 \\
6.39 \pm 0.03\end{array}$ & $\begin{array}{c}\bar{D}+N \\
\bar{D}^{*}+N, \bar{D}+N \\
B+N \\
B^{*}+N, B+N\end{array}$ \\
\hline
\end{tabular}

repulsion and to increase the attraction of the three-hadron system, I only consider pentaquarks with a minimally exotic isospin, and with low spin.

b) Here the flavour is decomposed in an s-wave system of a spin $1 / 2$ baryon and two pseudoscalar mesons, except for the vectors $D^{*}$ and $B^{*}$ which are also considered.

c) I consider as candidates for narrow pentaquarks systems where one hadron is attracted by both other ones. The criterion is used to discriminate which hadrons are bound and which are repelled.

d) In the case of some exotic flavour pentaquarks, only a four-hadron-molecule or a five-hadron-molecule would bind. These cases are not detailed, because they are difficult to create in the laboratory.

e) Moreover, in the particular case where one of the three hadrons is a $\pi$, binding is only assumed if the $\pi$ is the central hadron, attracted both by the other two ones. The $\pi$ is too light to be bound by just one hadron 40 .

f) The masses of the bound states with a pion are computed assuming a total binding energy of the order of $10 \mathrm{MeV}$, averaging the binding energy of the $\Theta^{+}$and of the $D^{*-} p$ system in the molecular perspective. The masses of the other bound states are computed assuming a total binding energy of the order of $50 \mathrm{MeV}$, averaging the binding energies of the $\Xi^{--}$and of the new positive-parity $D_{S}$ mesons.

g) Here higher excitations are neglected (they would further increase the binding 
\begin{tabular}{|lllll} 
November 24, & 2018 & $16: 4$ & WSPC/INSTRUCTION & FILE
\end{tabular}

paris2004proceedings

The Pentaquarks in the Linear Molecular Heptaquark Model 5

energy). This results in an error bar of $\pm 30 \mathrm{MeV}$ for the mass. When one of the hadrons in the molecule is not listed by the Particle Data Group [52, its mass is extracted from a recent lattice computation $[53$, and the error bar is $\pm 100 \mathrm{MeV}$.

e) Although three-body decay channels are possible through quark rearrangement, their observation requires high experimental statistics. Only some of the different possible two-body decay processes are detailed here.

To conclude, this work has performed a systematic search of exotic-flavour pentaquarks, using the heptaquark, or linear three-body hadronic-molecule perspective. This perspective is the result of standard QM computations of pentaquarks and hepatquark masses and of hadron-hadron short-range interactions. A large number of new exotic flavour-pentaquarks are predicted in Tables 1, 2 and 3 together with their two-body decay channels. The systems with more than one heavy antiquark are very numerous and they are omitted here. It is interesting to remark that degenerate states are shared by Tables 2 and 3. Moreover, some new multiquarks may be easier to bind than the presently observed exotic pentaquarks.

\section{Acknowledgments}

I am grateful to Katerina Lipka, Achim Geiser, Paula Bordalo and Pedro Abreu for discussions on the possibility to detect new exotic pentaquarks. This talk is devoted to encourage the experimental search for new multiquarks.

\section{References}

1. R.L. Jaffe, SLAC-PUB-1774, talk presented at the Topical Conf. on Baryon Resonances, Oxford, England, July 5-9, 1976; R. L. Jaffe, Phys. Rev. D 15281 (1977).

2. D. Diakonov, V. Petrov and M. V. Polyakov, Z. Phys. A 359305 (1997) arXiv:hep-ph/9703373.

3. T. Nakano et al. [LEPS Collaboration], Phys. Rev. Lett. 91, 012002 (2003) arXiv:hep-ex/0301020.

4. V. V. Barmin et al. [DIANA Collaboration], Phys. Atom. Nucl. 66, 1715 (2003) [Yad. Fiz. 66, 1763 (2003)] arXiv:hep-ex/0304040.

5. S. Stepanyan et al. [CLAS Collaboration], Phys. Rev. Lett. 91, 252001 (2003) arXiv:hep-ex/0307018.

6. J. Barth et al. [SAPHIR Collaboration], arXiv:hep-ex/0307083

7. A. E. Asratyan, A. G. Dolgolenko and M. A. Kubantsev, arXiv:hep-ex/0309042

8. V. Kubarovsky et al. [CLAS Collaboration], Phys. Rev. Lett. 92, 032001 (2004) [Erratum-ibid. 92, 049902 (2004)] arXiv:hep-ex/0311046.

9. A. Airapetian et al. [HERMES Collaboration], Phys. Lett. B 585, 213 (2004) arXiv:hep-ex/0312044.

10. H. G. Juengst [CLAS Collaboration], arXiv:nucl-ex/0312019

11. A. Aleev et al. [SVD Collaboration], arXiv:hep-ex/0401024

12. J. Z. Bai et al. [BES Collaboration], arXiv:hep-ex/0402012

13. M. Abdel-Bary et al. [COSY-TOF Collaboration], arXiv:hep-ex/0403011

14. K. T. Knopfle, M. Zavertyaev and T. Zivko [HERA-B Collaboration], arXiv:hep-ex/0403020

15. P. Z. Aslanyan, V. N. Emelyanenko and G. G. Rikhkvitzkaya, arXiv:hep-ex/0403044 
\begin{tabular}{|lllll} 
November 24, & 2018 & $16: 4$ & WSPC/INSTRUCTION & FILE
\end{tabular}

paris2004proceedings

6 P. Bicudo

16. S. Chekanov et al. [ZEUS Collaboration], arXiv:hep-ex/0403051

17. C. Pinkenburg, arXiv:nucl-ex/0404001

18. Y. A. Troyan, A. V. Beljaev, A. Y. Troyan, E. B. Plekhanov, A. P. Jerusalimov, G. B. Piskaleva and S. G. Arakelian, arXiv:hep-ex/0404003

19. S. Raducci, P. Abreu, A. De Angelis, DELPHI note 2004-002 CONF 683, March 2004.

20. I. Abt et al. [HERA-B Collaboration], arXiv:hep-ex/0408048

21. C. Alt et al. [NA49 Collaboration], Phys. Rev. Lett. 92, 042003 (2004) arXiv:hep-ex/0310014.

22. H. G. Fischer and S. Wenig, arXiv:hep-ex/0401014

23. J. W. Price, J. Ducote, J. Goetz and B. M. K. Nefkens [CLAS Collaboration], arXiv:nucl-ex/0402006

24. [H1 Collaboration], arXiv:hep-ex/0403017

25. F. Csikor, Z. Fodor, S. D. Katz and T. G. Kovacs, JHEP 0311 (2003) 070 arXiv:hep-lat/0309090.

26. S. Sasaki, arXiv:hep-lat/0310014

27. T. W. Chiu and T. H. Hsieh, arXiv:hep-ph/0403020

28. T. W. Chiu and T. H. Hsieh, arXiv:hep-ph/0404007

29. N. Mathur et al., arXiv:hep-ph/0406196

30. F. Okiharu, H. Suganuma and T. T. Takahashi, arXiv:hep-lat/0407001

31. F. Csikor, Z. Fodor, S. D. Katz and T. G. Kovacs, arXiv:hep-lat/0407033

32. N. Ishii, T. Doi, H. Iida, M. Oka, F. Okiharu and H. Suganuma, arXiv:hep-lat/0408030

33. C. Alexandrou, G. Koutsou and A. Tsapalis, arXiv:hep-lat/0409065

34. M. Mattson et al. [SELEX Collaboration], Phys. Rev. Lett. 89, 112001 (2002) arXiv:hep-ex/0208014.

35. L. Schmitt, S. Paul, R. Kuhn and M. A. Moinester, arXiv:hep-ex/0310049

36. J. M. Richard, arXiv:hep-ph/0212224

37. K. Cheung, arXiv:hep-ph/0308176

38. M. F. M. Lutz and E. E. Kolomeitsev, arXiv:nucl-th/0402084

39. M. A. Nowak, M. Rho and I. Zahed, Phys. Rev. D 48, 4370 (1993) arXiv:hep-ph/9209272.

40. P. Bicudo and G. M. Marques, Phys. Rev. D 69, 011503 (2004) arXiv:hep-ph/0308073.

41. F. J. Llanes-Estrada, E. Oset and V. Mateu, arXiv:nucl-th/0311020

42. T. Kishimoto and T. Sato, arXiv:hep-ex/0312003

43. P. Bicudo, arXiv:hep-ph/0403146

44. P. Bicudo, arXiv:hep-ph/0401106

45. P. Bicudo, arXiv:hep-ph/0403295

46. J. Wheeler, Phys. Rev. 52, 1083 (1937); ibidem 1107.

47. J. E. Ribeiro, Z. Phys. C 5, 27 (1980).

48. P. Bicudo, Phys. Rev. C 67, 035201 (2003).

49. P. Bicudo, S. Cotanch, F. Llanes-Estrada, P. Maris, J. E. Ribeiro and A. Szczepaniak, Phys. Rev. D 65, 076008 (2002) arXiv:hep-ph/0112015.

50. P. Bicudo, M. Faria, G. M. Marques and J. E. Ribeiro, Nucl. Phys. A 735, 138 (2004) arXiv:nucl-th/0106071.

51. P. Bicudo and J. E. Ribeiro, Phys. Rev. D 42, 1611 (1990); ibidem D 42, 1625; ibidem D 42, 1635.

52. K. Hagiwara et al. [Particle Data Group Collaboration], Phys. Rev. D 66, 010001 (2002).

53. N. Mathur, R. Lewis and R. M. Woloshyn, Phys. Rev. D 66, 014502 (2002) arXiv:hep-ph/0203253. 